\title{
Optical Lever Based Parylene Cantilevers for Biochemical Sensing
} \author{
Yongjun $\mathrm{Zhao}^{2}$, Qiao $\mathrm{Lin}^{3}$ and Yong $\mathrm{Xu}^{*}{ }^{* 1}$ \\ ${ }^{I}$ Electrical \& Computer Eng., Wayne State University, Detroit, MI 48202, USA \\ ${ }^{2}$ Carnegie Mellon University, USA \\ ${ }^{3}$ Columbia University, USA
}

Kuncheng Zheng ${ }^{1,}$, Waqas Khalid ${ }^{\S, 1}$, Zhuo Wang ${ }^{1}$, Yuefa Li $^{1}$, Rakesh B. Katragadda ${ }^{1}$,

\begin{abstract}
This paper reports the development of optical lever based parylene cantilevers for biochemical sensing. A simple but effective process to fabricate parylene cantilevers using Tetramethylammonium Hydroxide (TMAH) bulkmicromachining was demonstrated. A scheme of using anchoring holes was employed to prevent the peeling off of parylene during TMAH etching. Moreover, ridge structures were incorporated on the cantilever tip to achieve a flat surface for a well-controlled reflected laser spot. An analytical model was presented to guide the design of the cantilever sensor. The stability of the parylene cantilever sensor in water and buffer solution was demonstrated. Based on optical lever method, the parylene cantilevers successfully detected vapour phase alkanethiols, and DNA molecules in buffer solution.
\end{abstract}

Keywords: Biochemical sensors, cantilevers, parylene, surface stress.

\section{INTRODUCTION}

Biosensors play crucial roles in disease diagnosis, drug discovery, national security, environment monitoring, food safety, etc. Microfabricated cantilevers have proven to be a promising label-free sensing platform for a large variety of biomolecules [1-4]. Cantilever sensors can operate in either resonant mode (resonant frequency shift) or static mode (static bending). Because of its ability to operate in both liquid and air, the static mode operation is becoming especially attractive [5-19]. The enthusiasm on such kind of sensor is mainly because it has advantages such as label-free detection, simple sensor structure, high sensitivity, etc. The operation principle is schematically illustrated in Fig. (1). One side of the micro-cantilever is first immobilized with a probe or receptor layer (e.g., probe single-stranded DNA). The selective binding of target molecules (e.g., complementary single-stranded DNA) to the immobilized probe layer results in a surface stress change and as a consequence causes a mechanical bending of the cantilever. Therefore, the presence of target molecules can be detected by monitoring the bending of the cantilever. The bending of the cantilever is usually detected by optical lever method as shown in Fig. (1). With this method, the small deflection of the cantilever is converted to a much larger displacement on the position sensitive device by the reflected laser beam.

The displacement of the cantilever tip can be derived from Stoney's equation [20]:

\footnotetext{
*Address correspondence to this author at the Electrical \& Computer Eng., Wayne State University, Detroit, MI 48202, USA; Tel: 313-577-3850;

E-mail: yxu@ece.eng.wayne.edu

${ }^{\S}$ The first two authors contribute equally to this paper.
}

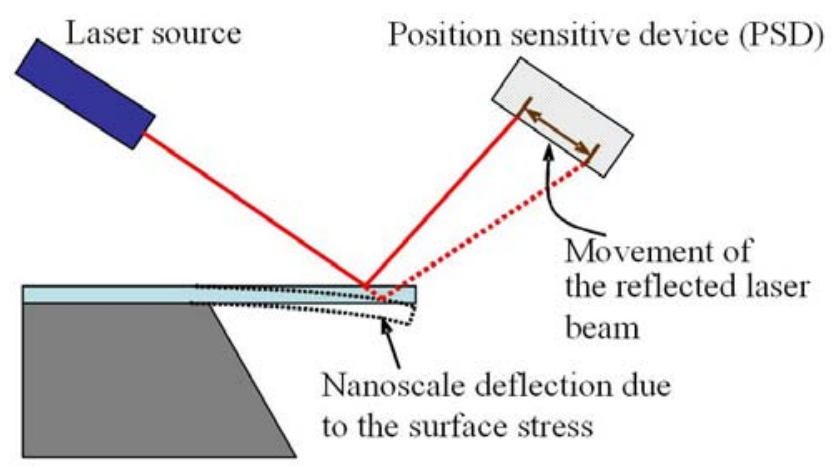

Fig. (1). Schematic presentation of the operating principle of cantilever biosensors. Note the deflection of the cantilever is exaggerated.

$\Delta y=\frac{3(1-v) L^{2}}{E t^{2}} \Delta \sigma$,

where $v$ is the Poisson's ratio, $E$ is the Young's modulus of cantilever material, $t$ is the cantilever thickness, $L$ is the cantilever length, and $\Delta \sigma$ is the surface stress change caused by the adsorbed molecules. It is apparent that the bending is inversely proportional to the Young's modulus of the cantilever material. So far, the majority of cantilever biosensors are fabricated using solid-state materials such as silicon nitride or silicon. If a softer material is employed, the deflection of cantilever will be more significant and thus the sensitivity will be much higher. Polymer cantilevers using SU-8 and other plastics have been demonstrated [21-24]. This paper reports the development of a cantilever biosensor based on parylene material. Parylene is the generic name for members of a unique family of thermoplastic polymers that are deposited by using the dimer of para-xylylene (di-paraxylylene, or DPXN) [25]. Parylene deposition is a room temperature, highly conformal, vapor phase process. 
Parylene is chemically inert and is not attacked by most wet chemicals. Therefore, we can use wet chemicals such as TMAH (Tetramethylammonium Hydroxide) to fabricate parylene cantilevers with very low cost. There are several members of parylene commonly used, such as parylene $\mathrm{N}, \mathrm{C}$, $\mathrm{F}$, and D. Parylene $\mathrm{C}$ is chosen as the cantilever material because its deposition rate is fairly high $(3 \sim 5 \mu \mathrm{m} / \mathrm{hour})$. Parylene C has a Young's modulus of $2.8 \mathrm{GPa}$, two orders of magnitude smaller than that of silicon nitride.

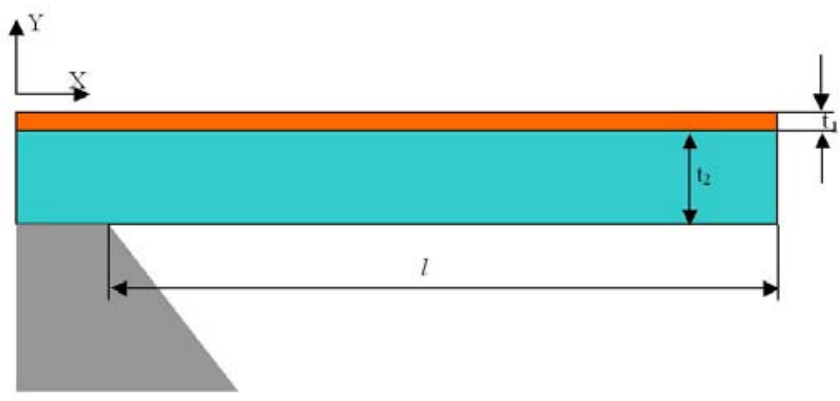

Fig. (2). Cross-sectional view of a cantilever sensor. The top layer is made of gold and the bottom layer is made of parylene $\mathrm{C}$.

\section{THEORETICAL CALCULATIONS}

To immobilize the probe layer, a metal layer such as gold is usually coated on the cantilever surface. Therefore, the cantilever sensor should be treated as a composite beam (bimetallic strip), which is composed of two different materials, as shown in Fig. (2). If we assume all the layers have the same width $w$ and length $l$, the neutral plane of the composite beam locates at [26]:

$y_{c}=\frac{\sum_{i} E_{i}^{*} t_{i} y_{c i}}{\sum_{i} E_{i}^{*} t_{i}}$

where $y_{c i}, t_{i}$ and $E_{i}^{*}$ are the coordinate of geometry centre, thickness and the modified Young's modulus of the $i^{\text {th }}$ layer $(\mathrm{i}=1,2)$, respectively. The modified Young's modulus can be expressed as $E_{i}^{*}=\frac{E_{i}}{1-v_{i}}$, where $E_{\mathrm{i}}$ and $v_{\mathrm{i}}$ are the Young's modulus and Poisson's ratio of the $\mathrm{i}^{\text {th }}$ layer respectively. The moment of inertia of the $\mathrm{i}^{\text {th }}$ layer is given by [31]:

$I_{z i}=\frac{w t_{i}^{3}}{12}+\left(y_{c}-y_{c i}\right)^{2} w t_{i}$

The bending angle of the tip can be written as [32]:

$\theta=-\frac{\sigma w\left(y_{t o p}-y_{c}\right) l}{\sum_{i} E_{i}^{*} I_{z i}}$

where $\sigma$ is the surface stress and $y_{\text {top }}$ is the coordinate of the top surface (gold). The displacement of the cantilever tip for small bending angle thus can be written as:

$$
\Delta y \approx \frac{l}{2} \theta=-\frac{\sigma w\left(y_{t o p}-y_{c}\right) l^{2}}{2 \sum_{i} E_{i}^{*} I_{z i}}
$$

If we have only one layer, the above equation will be degenerated to the well-known Stoney's equation. Based on this analytical model, it is observed that the metal layer, although very thin, has a non-negligible impact on the sensitivity of the cantilever. For example, if the parylene is $2.6 \mu \mathrm{m}$, a $20 \mathrm{~nm}$ gold layer may reduce the sensitivity by $45 \%$.

For such a composite beam, if the temperature increases the beam will bend due to different linear thermal expansion coefficients of the two materials. If the temperature increment is $\Delta T$ and linear thermal expansion coefficients for two layers are $\gamma_{1}$ and $\gamma_{2}$ respectively, the tip displacement is given by Eq. 6 [27]. The typical value for the cantilever we used is in the range of $200 \sim 300 \mathrm{~nm} /{ }^{\circ} \mathrm{C}$.

$$
\Delta y_{t}=\frac{3\left(\gamma_{2}-\gamma_{1}\right) \Delta T\left(t_{1}+t_{2}\right) l^{2}}{t_{2}^{2}\left(4+6 \frac{t_{1}}{t_{2}}+4\left(\frac{t_{1}}{t_{2}}\right)^{2}+\frac{E_{1}^{*}}{E_{2}^{*}}\left(\frac{t_{1}}{t_{2}}\right)^{3}+\frac{E_{2}^{*}}{E_{1}^{*}} \frac{t_{2}}{t_{1}}\right)}
$$

\section{FABRICATION}

Parylene cantilevers have been fabricated using surface micromachining previously [28]. In this work a simple and low cost process to fabricate parylene $\mathrm{C}$ cantilevers using TMAH bulk-micromachining was demonstrated. A brief fabrication process is described in Fig. (3).

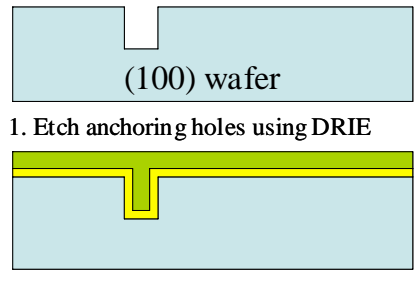

2. Grow $0.5 \mu \mathrm{m}$ thermal oxide and deposit a thin layer of parylene $\mathrm{C}$

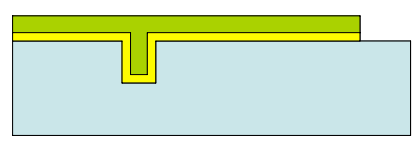

3. Pattern pary lene and oxide

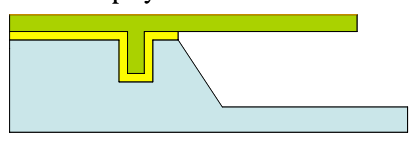

4. Free pary lene cantilever by $\mathrm{TMAH}$, strip oxide, and coat $\mathrm{Au} / \mathrm{Cr}$
Fig. (3). Process flow of parylene cantilevers.

It has been observed that TMAH does not etch parylene C. However, it attacks the interface between silicon and parylene and peels off parylene films. A scheme of using anchoring holes was employed to prevent the peeling off of parylene C during TMAH etching. Five-inch, (100) siliconwafers were used for the fabrication of our biosensors. The first step was to etch 20-30 $\mu \mathrm{m}$ deep anchor holes in the wafer using Deep Reactive Ion Etching (DRIE). In the second step, $0.5 \mu \mathrm{m}$ thick oxide layer was grown on the wafer using wet thermal oxidation. In the following step, a parylene C layer with desired thickness was vapour-phase deposited. The parylene $\mathrm{C}$ layer was then patterned using Reactive Ion Etching (RIE) with oxygen plasma. The silicon dioxide layer was removed from the openings using Buffered Oxide Etch (BOE). In the next step, TMAH was used to etch silicon, creating the freestanding cantilevers. The silicon underneath the cantilever was removed due to the convex corner undercut [29]. The oxide layer on the bottom surface of the cantilever was then stripped using BOE. Finally, a $20 \mathrm{~nm}$ gold layer was evaporated on the surface to provide a functional layer to immobilize DNA. Later the wafer was diced into small chips that were tested in this research.

It is also very interesting to note that after TMAH etching, the Parylene $\mathrm{C}$ becomes more hydrophobic. As illus- 
trated in Fig. (4), the contact angle was increased from $70^{\circ}$ to $90^{\circ}$.

(a)
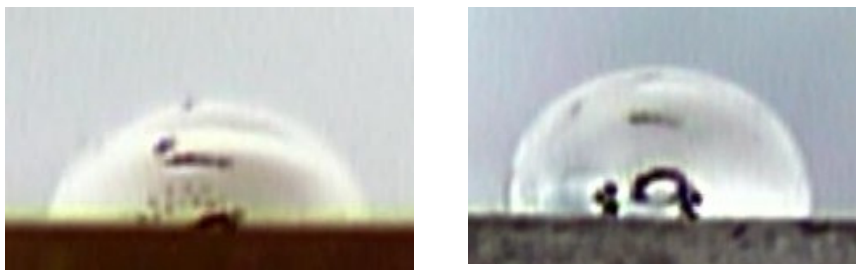

Fig. (4). Contact angle change on parylene $\mathrm{C}$ before (a) and after (b) 12 hours, $10 \%$ TMAH etching.

The SEM picture of two parylene cantilevers coated with a thin gold layer is shown in Fig. (5a). The anchoring holes are $50 \mu \mathrm{m} \times 50 \mu \mathrm{m}$. We found out stiction was a yield issue for this bulk-micromachining process. As shown in Fig. (5b), many long cantilevers were pinned to the substrate and the sidewall due to stiction.

This issue can be resolved using diluted TMAH $(5 \% \sim 10 \%)$, which results in very rough etched silicon surface [30]. By using 10\% TMAH, the stiction was reduced significantly as shown in Fig (6a). The resulted rough silicon surface is shown in Fig. (6b). The longest freestanding cantilever fabricated was $750 \mu \mathrm{m}$. As a comparison, when etched with $25 \% \mathrm{TMAH}$, the longest freestanding cantilever was only $350 \mu \mathrm{m}$.

As shown in Fig. (6), the cantilevers fabricated using this technique usually curled up due to stress gradient, making it difficult to obtain a good reflected laser spot from the tip of the cantilever. To address this issue, cantilever tips were integrated with ridge structures, which were formed by filling trenches etched on the cantilever tip areas. The trenches were etched together with the anchoring holes using DRIE in the very first step. Therefore the fabrication process was not complicated. These trenches were $4 \mu \mathrm{m}$ wide and 50-100 $\mu \mathrm{m}$ long and about $20-30 \mu \mathrm{m}$ deep. Since parylene coating is conformal, it completely filled the trenches, leading to planarized surfaces for photolithography in the following steps. A brief fabrication process of the new cantilevers is described in Fig. (7).

Fig. (8) shows the SEM pictures of cantilevers with ridges integrated on their tips. It can be observed that the ridges resulted in very flat cantilever tips, which can lead to very good reflected laser spot. It is worth noting that these ridges also help to reduce stiction by reducing contact areas. (a)

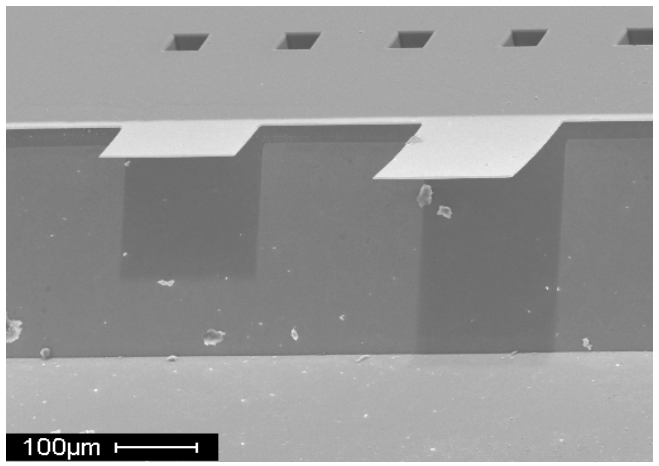

(b)

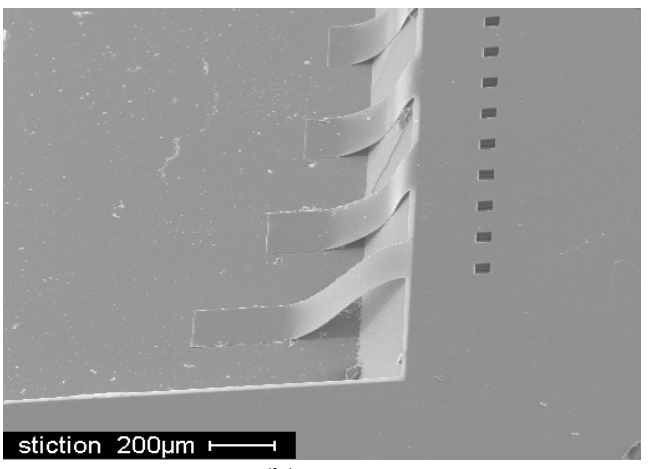

Fig. (5). (a) Scanning Electron Microscope (SEM) picture of gold-coated parylene cantilevers. The two cantilevers are $200 \mu \mathrm{m}$ wide, $2.2 \mu \mathrm{m}$ thick, $100 \mu \mathrm{m}$ and $200 \mu \mathrm{m}$ long, respectively. (b) SEM pictures of longer cantilevers $(720 \mu \mathrm{m}, 650 \mu \mathrm{m}, 550 \mu \mathrm{m}$ and $450 \mu \mathrm{m})$ sticking to the substrate.

(a)

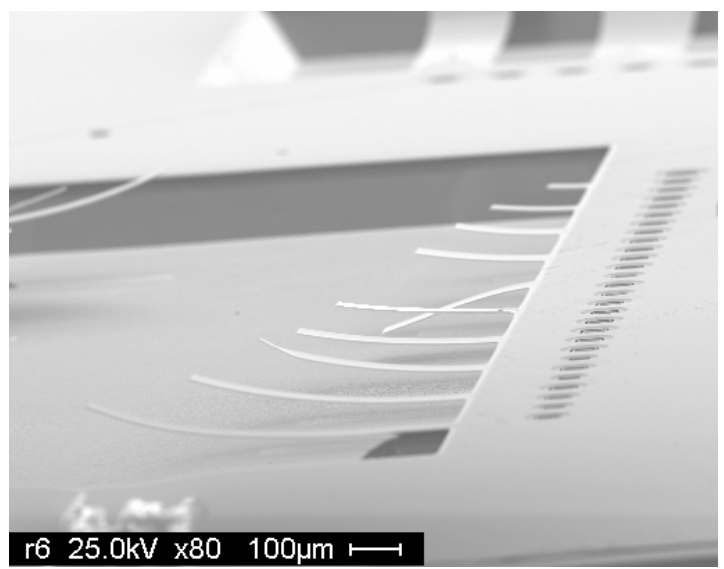

(b)

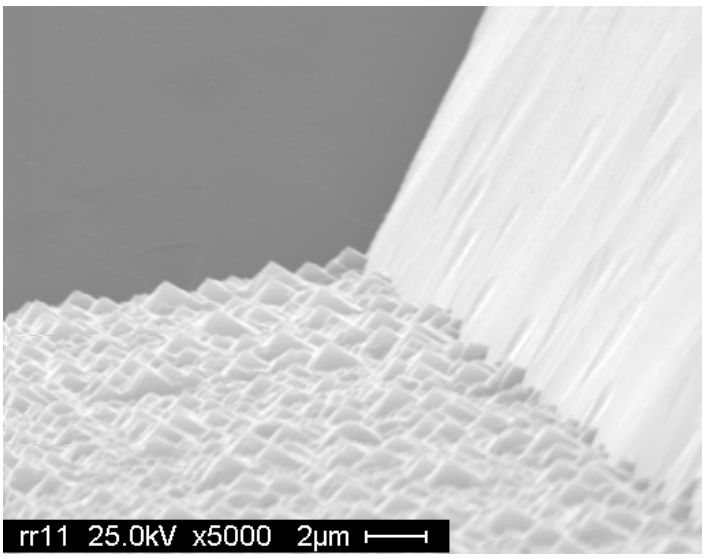

Fig. (6). (a) SEM picture of an array of cantilevers with lengths ranging from a $100 \mu \mathrm{m}$ to $720 \mu \mathrm{m}$. The upward curling of the cantilevers is obvious. (b) Rough surface resulted from diluted TMAH etching of silicon. 
Trenches at the tip

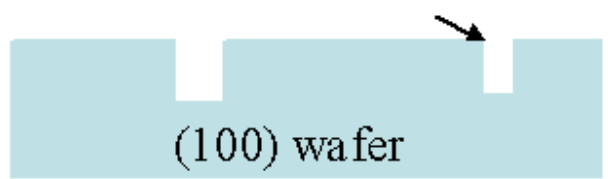

1. Etch anchoring holes and trenches using DRIE

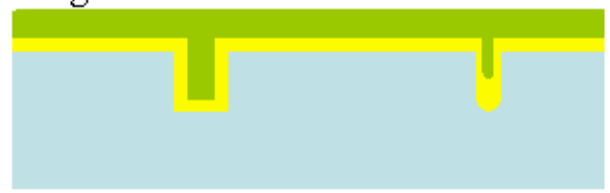

\section{Grow $0.5 \mu \mathrm{m}$ thermal oxide and deposit a thin lay er of parylene $\mathrm{C}$}

Fig. (7). Process flow of parylene cantilevers with trenches at the tip.

\section{EXPERIMENTAL}

A laser diode with fibre pigtail $(635 \mathrm{~nm}$ wave length, ThorLabs, NJ, USA) was used to measure the deflection of the cantilever. The reflected laser beam from the cantilever was monitored using a PSD (position-sensing device, model name: PSM 2-10, On-trak Photonics Inc, CA, USA). The PSD has a sensing area of $10 \mathrm{~mm} \times 10 \mathrm{~mm}$ and a resolution of $250 \mathrm{~nm}$. The output of the PSD was amplified by position sensing amplifier (OT- 301, On-trak Photonics Inc, CA, USA) and recorded using a DAQ card (PCI - 6250 with 16 bit resolution, National Instruments, Austin, TX, USA). One thousand samples were collected in every second. Every data point was calculated by averaging the 1000 samples within one second. A polysilicon thermistor was used to record the ambient temperature change. In addition, a thermal couple was placed inside the fluid cell to monitor the temperature difference between the solution and ambient. It is worth noting that it is crucial since the solution and the ambient may have different temperatures. The whole setup was enclosed in a black box on a vibration-isolated table to avoid noises from background light and ground vibration. The cantilevers

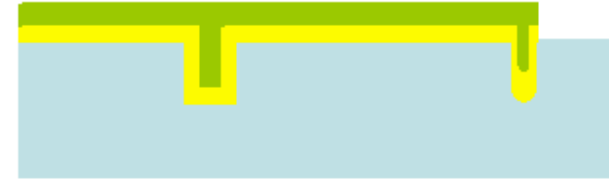

\section{Pattern parylene and oxide}

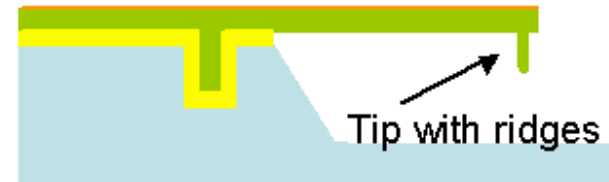

\section{Free parylene cantilever by \\ $\mathrm{TMAH}$, strip oxide, and coat $\mathrm{Au} / \mathrm{Cr}$}

used in experiments have a dimension of $375 \mu \mathrm{m} \times 200 \mu \mathrm{m}$ $\times 2.65 \mu \mathrm{m}$ and are coated with $20 \mathrm{~nm}$ gold on the top surface, if not specified.

\subsection{Temperature Sensitivity Test}

The fluid cell with cantilever sensors was placed on a Peltier heater to measure the temperature sensitivity. The change in the position of the tip of the cantilever along with the change in the temperature of DI water is plotted in Fig. (9). The temperature sensitivity of the parylene cantilever was measured to be $\sim 240 \mathrm{~nm} /{ }^{\circ} \mathrm{C}$. This is comparable to the theoretical bending of the tip of the cantilever $\left(\sim 227 \mathrm{~nm} /{ }^{\circ} \mathrm{C}\right)$ calculated using Eq. 6, assuming coefficients of thermal expansions (CTEs) are $14 \times 10^{-6} / \mathrm{K}$ for gold and $69 \times 10^{-6} / \mathrm{K}$ for parylene. As the temperature increased, the cantilever bent upward because parylene $\mathrm{C}$ has a higher thermal coefficient of expansion than gold. It can be observed that the temperature sensitivity is not negligible. Therefore, the temperature needs to be carefully monitored during the operation in order to detect low concentrations of biomolecules.

(b)

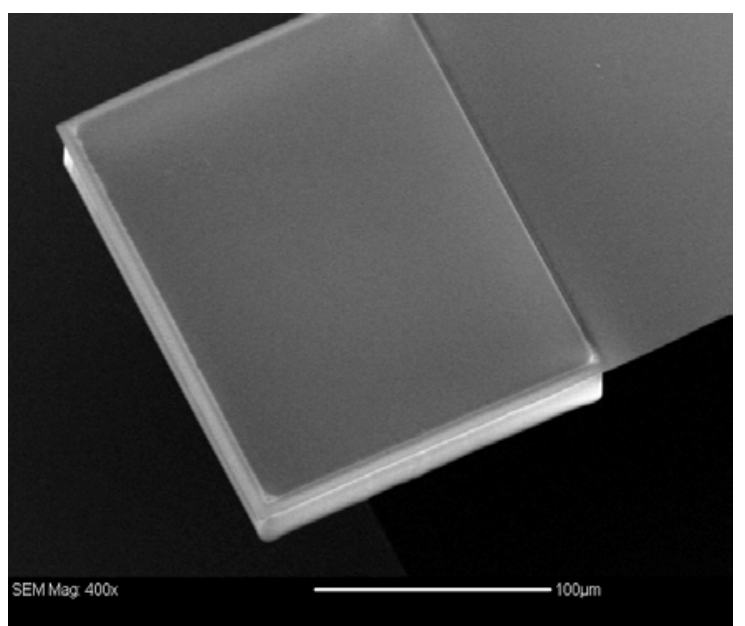

Fig. (8). (a) SEM picture of a gold-coated parylene cantilever with two parallel ridges on both sides of the tip. (b) SEM picture of a goldcoated parylene cantilever with rectangular ridge under the tip. The scale bars on figures (a) and (b) are $200 \mu \mathrm{m}$ and $100 \mu \mathrm{m}$, respectively. 


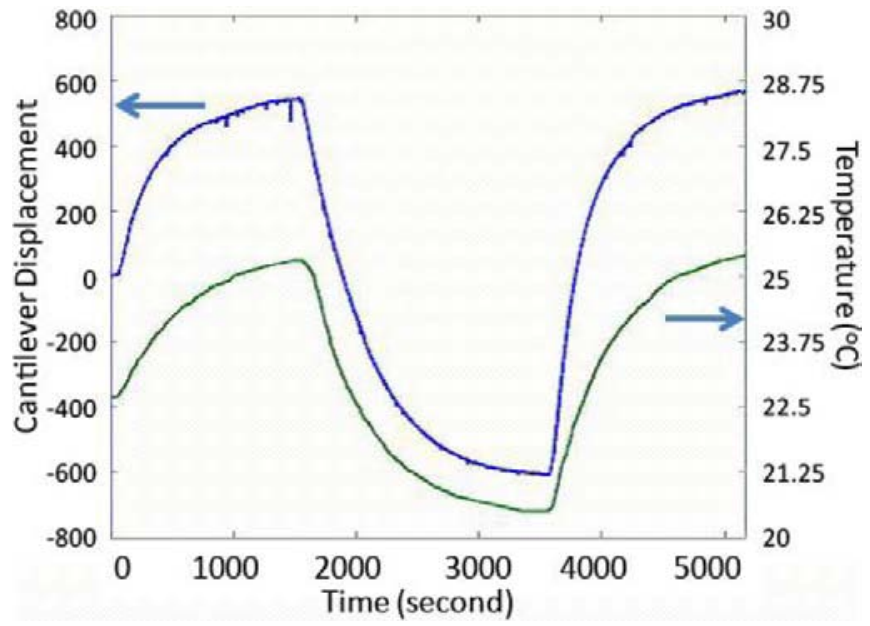

Fig. (9). Temperature sensitivity of a cantilever sensor. $20 \mathrm{~nm}$ of gold is coated on the surface of the cantilever.

\subsection{Stability of Parylene Cantilever}

Stability of parylene cantilevers in DI water was studied. Fig. (10a) shows the cantilever deflection and temperature change of the solution over a period of more than one hour. It can be clearly observed that the displacement of the cantilever follows the same trend as the temperature. As shown in Fig. (10b), after temperature compensation (using the temperature sensitivity measured in section 4.1), the cantilever became very stable with a standard deviation of $3.3 \mathrm{~nm}$.

\subsection{Sensitivity to PBS Concentration}

We studied the sensitivity of gold-coated parylene cantilevers to PBS concentration. The result of the experiment is shown in Fig. (11). The chip with cantilever sensors was placed in the fluid cell, which was filled completely with DI water. Then the laser was focused on a cantilever and the bending of the cantilever was monitored using a Photo Sensing Diode (PSD). DI water and PBS solution with a concentration of $1 \mathrm{X}$ were injected into the fluid cell in turn. Two consecutive injections were separated by 12 minutes. The ambient temperature measured by the polysilicon piezoresis- tor and the temperature difference between the solution and ambient are in Fig. (11a). This result clearly shows that the ambient temperature and solution temperature are not necessarily same. The displacement of the cantilever was monitored by the PSD and shown in Fig. (11b). The temperature of the solution is also plotted in Fig. (11b), by combining results of polysilicon thermistor and thermal couple together. The temperature-compensated displacement is shown in Fig. (11c). From this experiment, we found that the displacement of gold-coated parylene cantilever caused by the change of buffer concentration is negligible after temperature compensation. This result again signifies the importance of monitoring the solution temperature in situ. It is worth noting that this conclusion will not be valid if the cantilever surface is immobilized with some biomolecules such as DNA.

\subsection{Alkanethiol Test}

A cantilever sensor $(250 \mu \mathrm{m} \times 200 \mu \mathrm{m} \times 1.65 \mu \mathrm{m})$ was placed in an empty fluid cell $(5 \mathrm{ml}$ in volume) and the laser was focused on the tip to obtain a base line for about 50 minutes. Then 3 drops of 1-Octanethiol (98.5+\%, SigmaAldrich Inc, Mo, USA) were injected in the fluid cell. The volatile alkanethiol evaporated and immobilized on the gold surface, inducing surface stress on the cantilever. The recorded data for the displacement of the tip of the cantilever is shown in Fig. (12). According to the data recorded, the tip of the cantilever bends about $165 \mathrm{~nm}$ downwards (away form the gold surface). This bending corresponds to a compressive surface stress of about $0.030 \mathrm{~N} / \mathrm{m}$ according to Eq. 5 . The surface stress induced falls within the range reported in the literature $(0.001-0.250 \mathrm{~N} / \mathrm{m}$ depending on the alkanethiol carbon chain length and magnitude of surface coverage) [24].

The test was repeated with water drops instead of alkanethiol. The response of the gold-coated cantilever for the absorption of the water vapours is shown in Fig. (13). It can be clearly seen that there was no change in the position of the cantilever tip when water drops were added in the fluid cell. This further confirms that the displacement in Fig. (12) was caused by the alkanethiol molecules. (a)

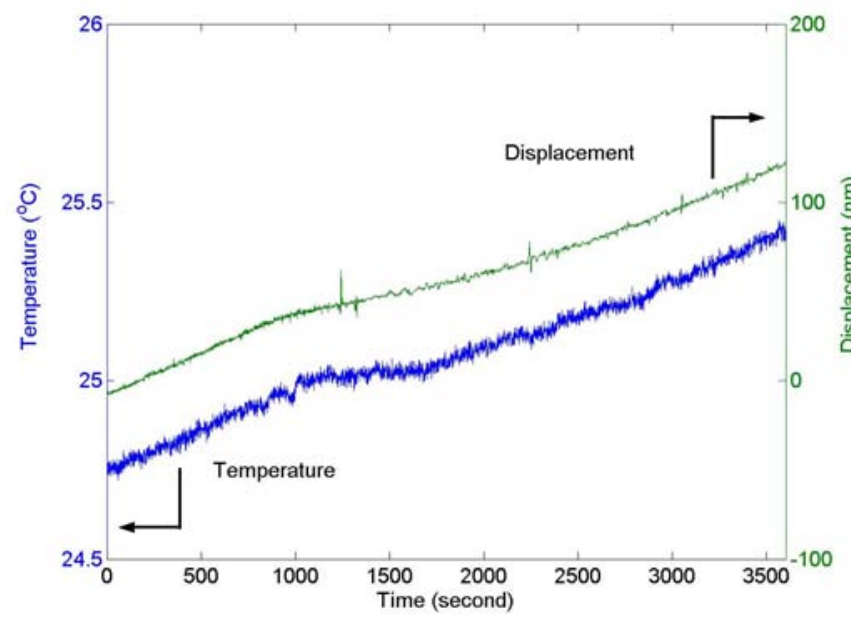

(b)

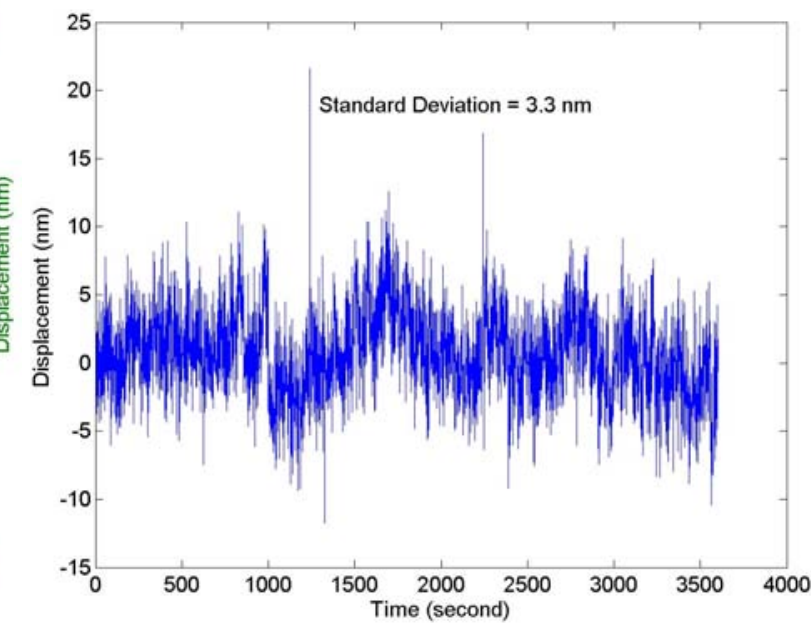

Fig. (10). (a) Temperature change and cantilever displacement versus time. (b) Temperature-compensated cantilever displacement. 
(a)

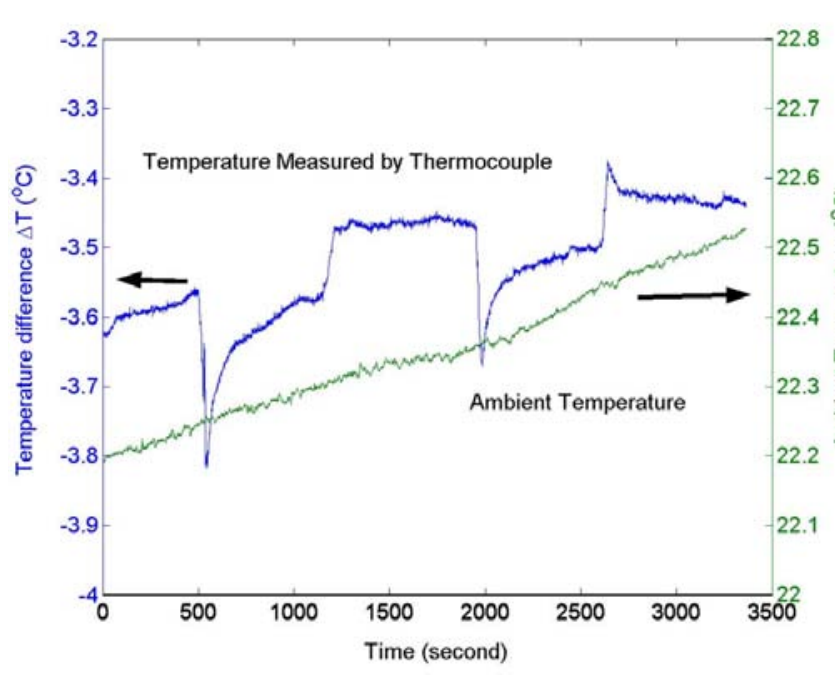

(b)

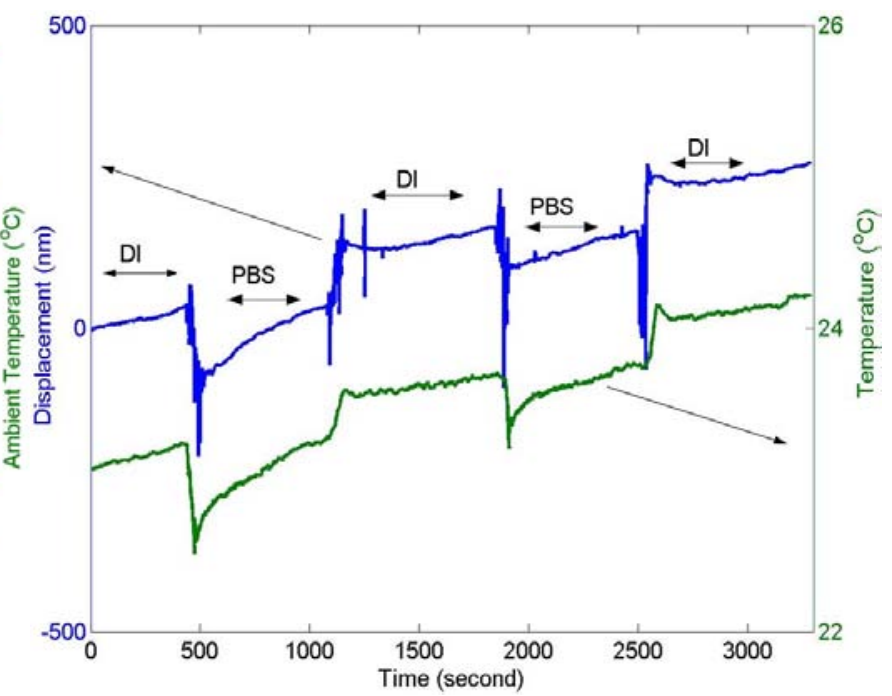

(c)

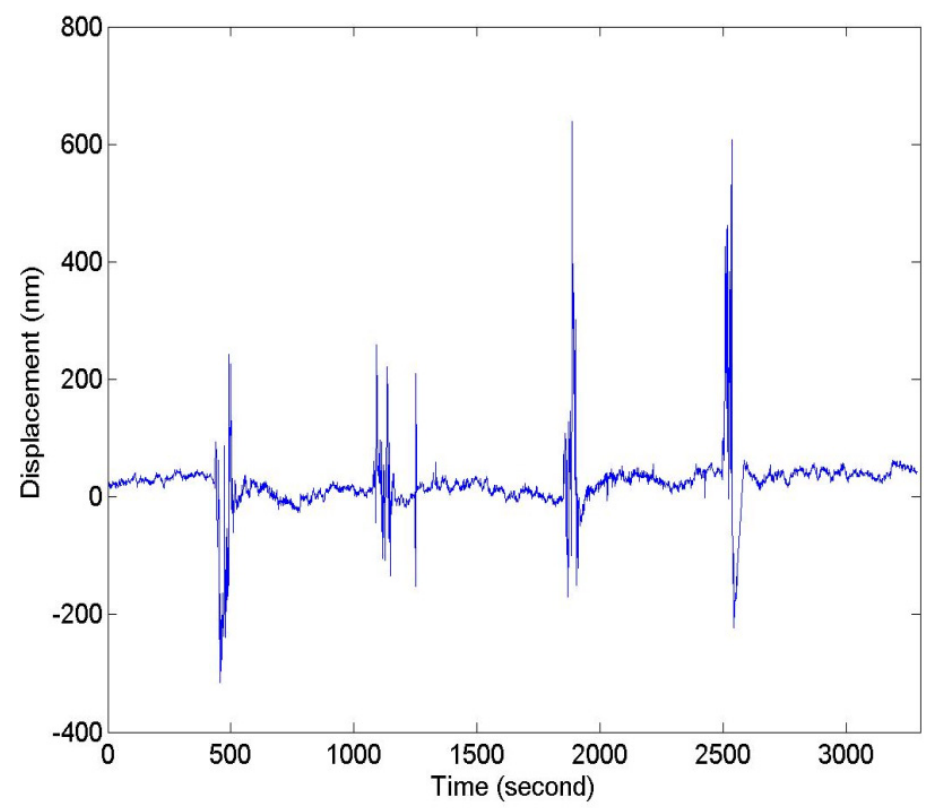

Fig. (11). The sensitivity of a parylene cantilever to the concentration of PBS. (a) Ambient temperature measured by polysilicon thermistor and the temperature difference between the solution and ambient measured by thermocouple. (b) Displacement of the cantilever tip and the temperature change of the solution when it was switched between DI water and 1X PBS. (c) Displacement of the cantilever tip after temperature-compensation.

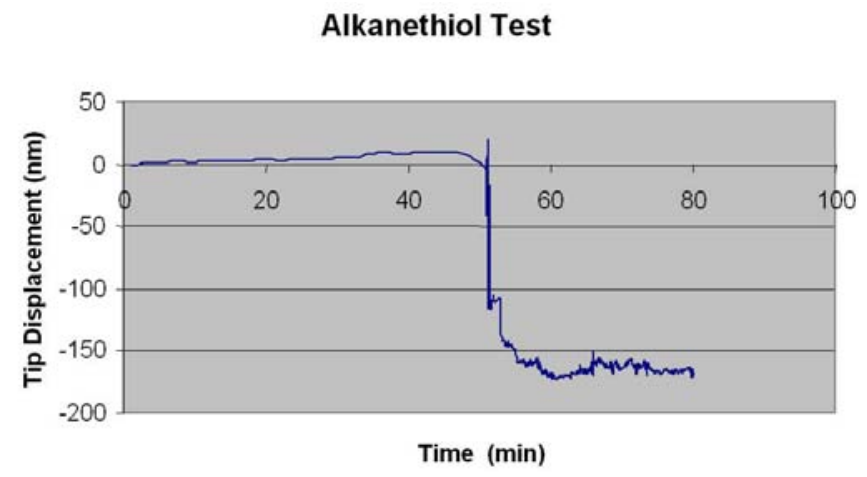

Fig. (12). Data showing the sensitivity of cantilever sensor to alkanethiol.

\subsection{DNA Test}

40-nt-long ssDNA nucleotide was used as the probe molecule (Synthegen, Florida). The sequence for the probe ssDNA molecule was 5'-TTAAGGTCTGGACTGGCCTG AATTTAGCGCCAGCTTACTG-3' and the sequence of the complementary target ssDNA was 3'-CAGACCTGACCG GAC-5' [14]. A parylene cantilever $(250 \mu \mathrm{m} \times 200 \mu \mathrm{m} \times 2.2 \mu \mathrm{m})$ was placed in the fluid cell and flushed with buffer solution. The probe molecules were mixed with the buffer solution and injected into the fluid cell $(\sim 8 \mu \mathrm{M})$. The deflection of the cantilever tip is shown in Fig. (14). According to the data recorded, the cantilever bent about $215 \mathrm{~nm}$ downwards (away from the gold surface). This shows a higher sensitivity of the parylene cantilever compared to its silicon nitride 
counterparts [14]. This bending corresponds to a compressive surface stress of about $0.040 \mathrm{~N} / \mathrm{m}$ according to Eq. 5 .

\section{Alkanethiol Test Repeated with Water}

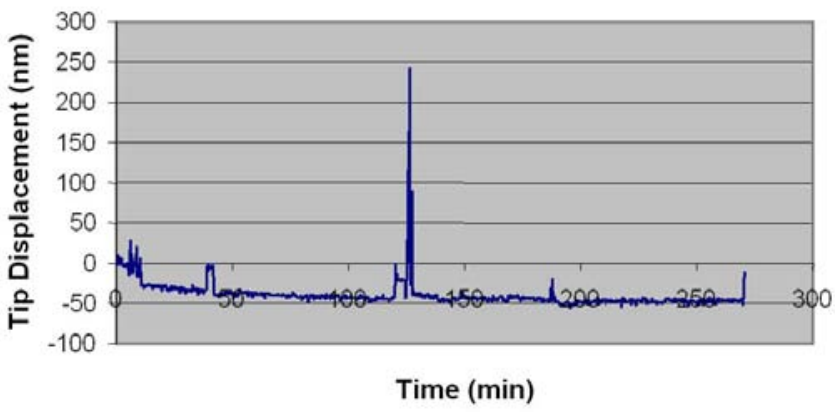

Fig. (13). Data showing the stability of cantilever sensor when water is injected instead of alkanethiol. The peak is the point when water drop was injected in the fluid cell.

\section{Immobalization of ssDNA}

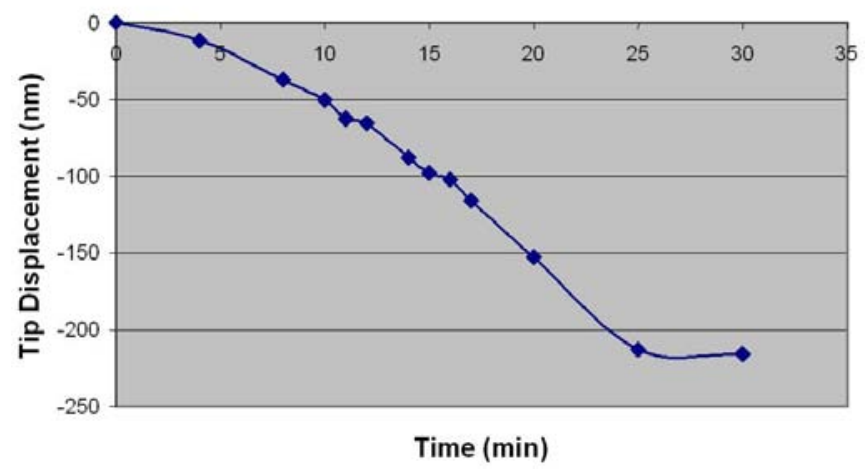

Fig. (14). The deflection of a parylene cantilever sensor $(250 \mu \mathrm{m} \times 200 \mu \mathrm{m} \times 2.2 \mu \mathrm{m})$ to the ssDNA immobilization on gold surface.

After 40 minutes, the fluid cell was flushed with the buffer solution. The target molecules were then mixed with the buffer solution $(\sim 3.75 \mu \mathrm{M})$ and injected into the fluid cell. Again, the deflection of the cantilever tip was monitored using the optical lever. The response of the parylene cantilever during the hybridization of complementary target ssDNA is shown in Fig. (15). According to the recorded data, the cantilever bent about $75 \mathrm{~nm}$ upwards (towards the gold surface). This means that hybridization reduces the compressive stress induced by the immobilization. This observation is consistent with the result reported in [14]. The $75 \mathrm{~nm}$ bending corresponds to a reduction in compressive surface stress of about $0.014 \mathrm{~N} / \mathrm{m}$ according to Eq. 5, leading to a sensitivity of $5.4 \mathrm{~nm} /(\mathrm{mN} / \mathrm{m})$. This again shows a higher tip displacement sensitivity of the parylene cantilever compared to its silicon nitride counterparts [14].

\section{CONCLUSION}

This paper presents development of parylene cantilever sensors. Parylene cantilevers were successfully fabricated using low-cost TMAH bulk-micromachining. Ridges were integrated to make flat cantilever tips for better reflected laser spots. The bimaterial cantilever sensor was stable in water and PBS, but was very sensitive to temperature. The high sensitivity of parylene cantilevers was demonstrated by alkanethiols and DNA sensing. The parylene cantilever shows a promising future as a powerful tool for the detection of chemicals and macro biomolecules.

\section{Hybridization of ssDNA}

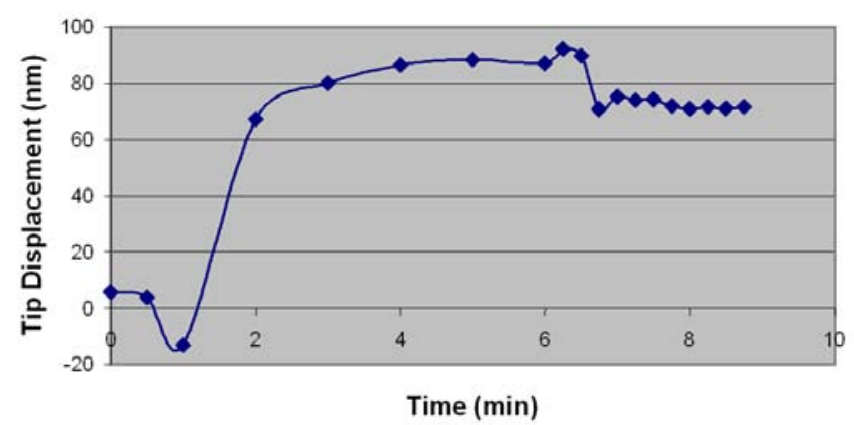

Fig. (15). The response of the cantilever sensor during the hybridization of target ssDNA with the probe ssDNA immobilized on the gold surface of the cantilever sensor.

\section{ACKNOWLEDGEMENTS}

The fabrication of the parylene cantilever was partly carried out in the SSIM cleanroom at Wayne State University. This research is supported by Wayne State University President's Research Enhancement Program.

\section{REFERENCES}

[1] Raiteri R, Grattarola M, Butt H, Skladal P. Micromechanical cantilever-based biosensors. Sens Actuators B Chem 2001; 79: 115-26.

[2] Lavrik N, Sepaniak M, Datskos P. Cantilever transducers as a platform for chemical and biological sensors. Rev Sci Instrum 2004; 75: 2229-53.

[3] Ziegler C. Cantilever-based biosensors. Anal Bioanal Chem 2004; 379: 946-59.

[4] Carrascosa L, Moreno M, Alvarez M, Lechuga M. Nanomechanical biosensors: a new sensing tool. Trac-Trends in Anal Chem 2006; 25: 196-206.

[5] Raiteri R, Nelles G, Butt G, Knoll W, Skladal P. Sensing of biological substances based on the bending of microfabricated cantilevers. Sens Actuators B Chem 1999; 61: 213-7.

[6] Fritz J, Baller M, Lang H, et al. Translating biomolecular recognition into nanomechanics. Science 2000; 288: 316-8.

[7] Hansen K, Ji H, Wu G, et al. Cantilever-based optical deflection assay for discrimination of DNA single-nucleotide mismatches. Anal Chem 2001; 73: 1567 -71.

[8] Grogan C, Raiteri R, O'Connor G, et al. Characterisation of an antibody coated microcantilever as a potential immuno-based biosensor. Biosens Bioelectron 2002; 17: 201-7.

[9] Arntz Y, Seelig J, Lang H, et al. Label-free protein assay based on a nanomechanical cantilever array. Nanotechnology 2003; 14: 8690.

[10] Pei J, Tian F, Thundat T. Glucose biosensor based on the microcantilever. Anal Chem 2004; 76: 292-7.

[11] Subramanian A, Oden P, Kennel S, et al. Glucose biosensing using an enzyme-coated microcantilever. Appl Phys Lett 2002; 81: 3857.

[12] Weeks B, Camarero J, Noy A, et al. A microcantilever-based pathogen detector. Scanning 2003; 25: 297-9.

[13] Wu G, Datar R, Hansen K, et al. Bioassay of prostate-specific antigen (PSA) using microcantilevers. Nat Biotechnol 2001; 19: 856-60.

[14] Wu G, Ji H, Hansen K, et al. Origin of nanomechanical cantilever motion generated from biomolecular interactions. Proc Natl Acad Sci USA 2001; 98: 1560-4. 
[15] McKendry R, Zhang J, Arntz Y, et al. Multiple label-free biodetection and quantitative DNA-binding assays on a nanomechanical cantilever array. Proc Natl Acad Sci USA 2002; 99: 9783-8.

[16] Yue M, Lin H, Dedrick D, et al. A 2-D microcantilever array for multiplexed biomolecular analysis. J Microelectromech Syst 2004; 13: 290-9.

[17] Savran C, Knudsen S, Ellington A, Manalis S. Micromechanical detection of proteins using aptamer-based receptor molecules. Anal Chem 2004; 76: 3194-8.

[18] Wee K, Kang G, Park J, et al. Novel electrical detection of labelfree disease marker proteins using piezoresistive self-sensing micro-cantilevers. Biosens Bioelectron 2005; 20: 1932-8.

[19] Huber F, Hegner M, Gerber C, Guntherodt H, Lang H. Label free analysis of transcription factors using microcantilever arrays. Biosens Bioelectron 2006; 21: 1599-605.

[20] Stoney G. The tension of metallic films deposited by electrolysis. Proc R Soc Lond Ser A 1909; 82: 172-5.

[21] Thaysen J, Yalcinkaya A, Vettiger P, Menon A. Polymer-based stress sensor with integrated readout. J Phys D Appl Phys 2002; 35 : 2698-703.

[22] Calleja M, Tamayo J, Nordstrom M, Boisen A. Low-noise polymeric nanomechanical biosensors. Appl Phys Lett 2006; 88: 1139013.
[23] Johansson A, Blagoi G, Boisen A. Polymeric cantilever-based biosensors with integrated readout. Appl Phys Lett 2006; 89: 1735057.

[24] McFarland A, Colton J. Chemical sensing with micromolded plastic microcantilevers. J Microelectromech Syst 2005; 14: 1375-85.

[25] Parylene Data Sheet. Specialty Coating System, Indianapolis.

[26] Weinberg M. Working equations for piezoelectric actuators and sensors. J Microelectromech Syst 1999; 8: 529-33.

[27] Young W, Budynas R. Roark's formulas for stress and strain, 7th ed. McGraw Hill, New York 2002.

[28] Yao T, Yang X, Tai Y. BrF3 Dry Release Technologies for Large Freestanding Parylene MEMS: International Conference on SolidState Sensors and Actuators (Transducers '01), Munich, Germany 2001

[29] Madou M. Fundamentals of Microfabrication. CRC. Press, Boca Raton, NY 1997.

[30] Tabata O, Asahi R, Funabashi H, Shimaoka K, Sugiyama S. Anisotropic etching of silicon in TMAH solutions. Sens Actuators A Phys 1992; 34: 51-7.

[31] Wang Z. A novel Lab-on-Tip nanomechanical platform for single molecule DNA sequencing. Masters' Thesis, Electrical Engineering, Wayne State University, Detroit, MI 2006.

[32] Zhang Y, Ren Q, Zhao Y. Modeling analysis of surface stress on a rectangular cantilever beam. J Phys D Appl Phys 2004; 37: 2140-5.

(c) Zheng et al.; Licensee Bentham Open.

This is an open access article licensed under the terms of the Creative Commons Attribution Non-Commercial License (http://creativecommons.org/licenses/by$\mathrm{nc} / 3.0 /$ ) which permits unrestricted, non-commercial use, distribution and reproduction in any medium, provided the work is properly cited. 